\title{
Mining Wrinkle-Patterns with Local Edge- Prototypic Pattern (LEPP) Descriptor for the Recognition of Human Age-groups
}

\author{
Md Tauhid Bin Iqbal and Oksam Chae* \\ Dept. of Computer Science and Engineering, Kyung Hee University (Global Campus), \\ Giheung-gu, Yongin-si, Gyeonggi-do, 17104, South Korea. \\ Email: \{tauhidiq, oschae\} @khu.ac.kr \\ * Corresponding Author
}

Received: 11 April 2018; Accepted: 15 May 2018; Published: 08 July 2018

\begin{abstract}
Human age recognition from face image relies highly on a reasonable aging description. Considering the disparate and complex face-aging variation of each person, aging description needs to be defined carefully with detailed local information. However, aging description relies highly on the appropriate definition of different aging-affiliated textures. Wrinkles are considered as the most discernible textures in this regard owing to their significant visual appearance in human aging. Most of the existing image-descriptors, however, fail short to preserve diverse variations of wrinkles, such as a) characterizing stronger and smoother wrinkles, appropriately, b) distinguishing wrinkles from nonwrinkle patterns, and c) characterizing the proper texturestructures of the pixels belonging to the same wrinkle. In this paper, we address these issues by presenting a new local descriptor, Local Edge-Prototypic Pattern (LEPP) with the notion that LEPP preserves different variations of wrinkle-patterns appropriately in representing the aging description. In the coding, LEPP sets prototypic restrictions for each neighboring pixel using their relation with center pixel when they belong to an inlying-edge, and utilize such restrictions, afterwards, to prioritize specific neighbors showing significant edge-signature. This strategy appropriately encodes the inlying edge structure of aging-affiliated textures and simultaneously, avoids featureless texture. We visualize the stability of LEPP in terms of its robustness under noise. Our experiments show that LEPP preserves discernible aging variations yielding better accuracies than the state-of-theart methods in popular age-group datasets.
\end{abstract}

Index Terms - Mining winkle, Age-group Classification, (LEPP), Aging-cue, Wrinkle-patterns, Noise, (ADIENCE), (GALLAGHER), (FACES).

\section{INTRODUCTION}

Human age recognition from face image has become an inseparable part of diversified human-centric computing applications, including surveillance monitoring, biometric authentication, and forensic arts [1,
2]. However, the task of age recognition is very challenging in practice due to the disparate and complex individual face-aging mechanism [3,4]. Since human aging can mainly be represented by the aging-affiliated appearance changes on the face, description of these changes is the key to recognize human age. Therefore, detail and fine-point definition of the discernible agingaffiliated textures is required for a reliable agingdescription.

Earlier works on anthropometric studies show that craniofacial growth and skin aging are two common changes that occur during human aging [1], [5]-[8]. Craniofacial growth refers to the gradual shape change in the head skull, while skin aging preserves the texture (appearance) change information associated with skin aging. Our focus is confined to skin aging due to its finerlevel texture definition in distinct aging-affiliated patterns, such as, wrinkle, furrow, and blemishes that are considered as significantly visible cues for human aging [9]-[11]. Visually, furrows and blemishes appear similarly as wrinkles; nevertheless, wrinkles appear mostly among these patterns in human faces and become more evident with gradual age progression. Hence, wrinkles are considered as the most discriminative visual cue in human aging [1], [12]-[15]. Motivated by this, we further analyze the appearance of wrinkle patterns in face images more precisely and found that wrinkles mainly appear in two ways, a) strong wrinkles, and b) smoother wrinkles. We also observe that texture-structure of a wrinkle-pixel is significantly different from a nonwrinkle pixel. We argue that incorporating such characteristics of wrinkle patterns in the feature-code is of great importance in recognizing particular age-class. Moreover, subtle intensity distortion may affect the structure of the pixels belonging to a continuing-wrinkle. Therefore, measures should also be taken against such distortion effect in preserving the appropriate structure of the pixels belonging to a continuing wrinkle.

In this work, we address the above issues with the proposed feature descriptor, Local Edge-Prototypic Pattern $(L E P P)$ in order to ensure a reliable description of aging from facial image. We observe that proposed descriptor achieves better accuracy than other existing 
methods in popular datasets in recognizing human age groups. Moreover, we visualize the comparative efficacy of LEPP against other descriptors under noise, exhibiting the robustness of LEPP in such condition.

\section{A. Related Works}

Active methods for age recognition can be divided into following categories: anthropometric model [16]-[18], aging manifold model [3], [19]-[21], deep learning methods [22]-[23], and appearance-based method [14], [24]-[28].

Classical anthropometric models utilize geometric relationships among facial components to represent the facial description. Since facial components are difficult to detect under appearance changes, anthropometric models often end up with low recognition results. Agingmanifold approaches represent face into low-dimensional aging manifolds in embedded subspace. However, these methods are global in nature, and therefore, may lose important micro-level aging information in practice. Recently deep-learning approaches have been applied to age classification task. Most of these methods require additional training data to generate age-model yielding complexity and external dependency to the networkstructure. Moreover, high performance of recent deepmethods is still somewhat dependent on computerhardware. Due to such limitations, there is a growing need for traditional feature descriptors that are fast to compute, memory efficient, and yet exhibiting good discriminability and robustness. Such feature descriptors fall in the category of appearance-based approaches. Roughly speaking, these methods mainly use imagefilters to extract micro-level texture and shape information of face. In particular, there are two directions in appearance-based methods: texture-based descriptor, and edge-based descriptor.

Among texture-based descriptors, Local Binary Pattern (LBP) [24]-[26] is the most popular one. LBP generates code from the intensity difference between a pixel and its neighboring pixels. LBP is advantageous in monotonic illumination but shows sensitivity towards the noise and non-monotonic illuminations [29]-[30].3 Moreover, because of this naive texture specific coding scheme, LBP code is often inconsistent in capturing the direction of the edge appropriately [27]-[28], which, in turn, makes the LBP code weak in wrinkle patterns, where the edges are most prominent. Conversely, edge-based descriptors such as Positional Ternary Pattern (PTP) [27], Local Directional Pattern (LDP) [29] and Local Directional Pattern (LDN) [30] use the position of top few responses of compass masks to denote the principal axes of the edge, and generate their codes using such edge-directional information. Although edge-based descriptors show their efficacy in preserving the principal direction of wrinkle (e.g., edge) properly, they fail short to preserve many other variations. For instance, owing to using small $3 \times 3$ image region, these descriptors gain limited information about the neighboring structure of the target pixel. Therefore, in case of dealing a smoother wrinkle, they often fail to understand the gradual texture change of such wrinkles and misinterpret them as other random textures. Moreover, using such a small region in generating code is prone to subtle noise and local distortion, which may deviate the principal direction-axes and generate different codes. Moreover, except some recent edge-descriptors, such as Directional AgePrimitive Pattern (DAPP) [28], most of the above methods generate codes for featureless textures, such as flat and random noisy textures, arising uncertainty in the feature description. Therefore, roughly speaking, existing descriptors still suffer to incorporate different characteristics of wrinkle patterns unambiguously in representing the age-description, which needs to be addressed properly in order to achieve better agerecognition results.

\section{B. Our Contribution}

In this paper, we address the aforementioned issues with a new local descriptor, Local Edge-Prototypic Pattern (LEPP), for the age-group recognition problem. The basic idea of LEPP is to set prototypic restrictions for the neighboring pixels using their relation with the center pixel in terms of the gradient information while both of them belong to an edge/line. LEPP uses these restrictions, afterwards, to prioritize the neighbors falling only in the direction of edge within the local neighborhood, ensuring the appropriate shape definition of different patterns, such as wrinkle, furrow, and blemish. Using gradient information allows LEPP to explore bigger neighboring region than that of the former edge-descriptors. This is advantageous to gain richer information of the texture structure, which, in turn, handles slight distortion-affect in local region ensuring consistent structures of the pixels. In this way, LEPP preserves different characteristics of wrinkle-patterns yielding a stable aging-definition. LEPP is found to achieve better performance than other descriptors in benchmark datasets, as well as, it performs well under noise. Hence, the contribution of this work can be summarized as,

1) We present a new local descriptor, Local EdgePrototypic Pattern (LEPP) that preserves different characteristics of aging-affiliated textures, especially, wrinkles, consistently, yielding a reliable aging-description.

2) We evaluate the stability of LEPP in terms of its robustness to noise, where LEPP is found to perform better than other current descriptors.

3) We evaluate the performance of proposed descriptor on benchmark age-group datasets, where LEPP outperforms existing descriptors convincingly. Moreover, LEPP achieves better accuracy than contemporary deep learning methods at specific case.

\section{METHODOLOGY}

Since the aging-affiliated textures, such as wrinkles, blemishes, and furrows mainly appear as edges in the image [14], [27]-[28], characterizing the signature of the 
edge is crucial in aging description. In our approach, we represent the direction of the inlying edge of a pixel by prioritizing its specific neighbors that form edge/line with the center pixel. For this, we define a set of structural restrictions for the surrounding neighbors. These restrictions are defined based on the prototypic gradient orientation of a neighbor pixel when it forms an edge with the center pixel. We prioritize the neighbors that show such gradient orientations and consider them as the token of the direction of inlying edge. Besides, the neighbors that do not comply with such orientations are ignored. In this way, the proposed coding scheme ensures appropriate representation of the direction of the edge (e.g., wrinkles), and ignores random patterns.

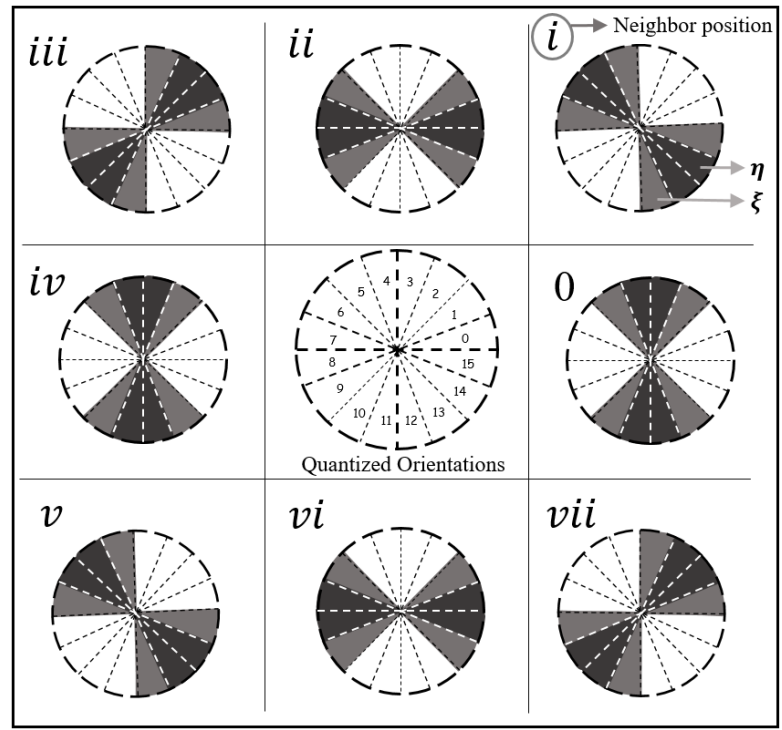

\begin{tabular}{|c|c|c|c|}
\hline \multirow{2}{*}{$\begin{array}{c}\text { Neighbor } \\
\text { Position }\end{array}$} & \multicolumn{3}{|c|}{ Gradient Orientations } \\
\cline { 2 - 4 } & $\begin{array}{c}\text { Higher } \\
\text { weight, } \boldsymbol{\eta}\end{array}$ & $\begin{array}{c}\text { Lower } \\
\text { Weight, } \boldsymbol{\xi}\end{array}$ & $\begin{array}{c}\text { No } \\
\text { Weight }\end{array}$ \\
\hline $\mathbf{0 , \boldsymbol { i } \boldsymbol { v }}$ & $3,4,11,12$ & $2,5,10,13$ & Others \\
\hline $\boldsymbol{i}, \boldsymbol{v}$ & $5,6,13,14$ & $4,7,12,15$ & Others \\
\hline $\boldsymbol{i i}, \boldsymbol{v i}$ & $0,15,7,8$ & $1,14,6,9$ & Others \\
\hline $\boldsymbol{i i i}, \boldsymbol{v i i}$ & $1,2,9,10$ & $0,3,8,11$ & Others \\
\hline
\end{tabular}

Fig.1. Prototypic-orientations for each neighboring pixel are shown through black and gray colors. Orientations with black and gray colors denote higher $(\eta)$ and lower weight $(\xi)$, respectively. Assignment of $\eta$ and $\xi$ for each neighbor are also given explicitly in the associated table.

\section{A. Coding Scheme}

We initiate LEPP code computation by extracting gradient magnitude and orientation at each pixel of an image by applying $3 \times 3$ Sobel Operator. Let us denote an image pixel as $i$. We convolve Horizontal $(H)$ and vertical $(V)$ Sobel operators with that pixel to get corresponding horizontal and vertical edge responses $G_{i}^{H}$ and $G_{i}^{V}$ respectively,

$$
\begin{gathered}
G_{i}^{H}=H^{*} i, \\
G_{i}^{V}=V * i,
\end{gathered}
$$

where, $H$ and $V$ are defined as,

$$
\begin{aligned}
& H=\left[\begin{array}{ccc}
-1 & 0 & +1 \\
-2 & 0 & +2 \\
-1 & 0 & +1
\end{array}\right], \\
& V=\left[\begin{array}{ccc}
-1 & -2 & -1 \\
0 & 0 & 0 \\
+1 & +2 & +1
\end{array}\right] .
\end{aligned}
$$

We use $G_{i}^{H}$ and $G_{i}^{V}$ to compute gradient magnitude $G_{i}$ and gradient orientation $O_{i}^{q}$ of the pixel, $i$, by

$$
\begin{gathered}
G_{i}=\sqrt{\left(G_{i}^{H}\right)^{2}+\left(G_{i}^{V}\right)^{2}}, \quad \alpha\left(G_{i}\right)= \begin{cases}G_{i}, & \text { if }, G_{i}>\gamma \\
0, & \text { otherwise }\end{cases} \\
O_{i}^{q}=\tan ^{-1}\left(G_{i}^{V} / G_{i}^{H}\right),
\end{gathered}
$$

where, $\alpha\left(G_{i}\right)$ applies a threshold, $\gamma$, on the magnitude, $G_{i}$. This threshold filters the low magnitude of the featureless flat pixels that do not contribute to the representation of edge structure. Gradient magnitudes, that do not pass the threshold, $\gamma$, are set to zero, and pixels having such low magnitudes are not considered in the calculation of LEPP code, afterwards. In eq (6), the superscript, $q$, on orientation, $O_{i}$, denotes that $O_{i}$ is quantized into $q$ bins. We use $q=16$ in this work. We now calculate $\alpha\left(G_{i}\right)$ and $O_{i}^{q}$ for all the pixels of an input image.

We characterize the direction of the inlying edge by prioritizing the neighbors that form edge/line with center pixel. When an edge/line is formed between a neighbor pixel and the center pixel, gradient orientation at that neighbor appears orthogonal to the direction of that edge/line [31]-[32]. We regard these orientations as prototypic-orientations of the corresponding neighbors, which are marked by black colors in Fig. 1. In the coding, we impose higher preference to the neighbors showing such orientations, and consider them as the possible directions of the inlying edge. A neighbor usually does not show prototypic-orientations in the absence of an edge/line formed with center pixel, and hence we ignore the neighbors not showing the prototypic-orientation. Formally, we put weights at each $k^{\text {th }}$ neighbor based on its orientation $O_{i}^{q}$ as following, 


$$
\beta\left(O_{k}^{q}\right)= \begin{cases}\eta, & \text { if }, O_{k}^{q} \in\{a, b, c, d\} \\ \xi, & \text { if }, O_{k}^{q} \in\{p, q, r, s\} \\ 0, & \text { otherwise, }\end{cases}
$$

where, $\beta\left(O_{i}^{q}\right)$ returns higher weight value, $\eta$, when $O_{k}$ directly matches with any of its the predefined prototypic-orientations $(a, b, c, d)$. Besides, orientation appearing in the nearby bins $(p, q, r, s)$ of the prototypicorientations are assigned slightly lower weight, $\xi$. Assignments of $\eta$ and $\xi$ for each neighboring pixel can be found in the table given in Fig. 1. It is noteworthy to mention that we use Gaussian weighting for setting the weight values of $\eta$ and $\xi$. We now prioritize the neighbors by computing priority-score, $P S_{k}$, at each $k^{\text {th }}$ neighbor,

$$
\mathrm{PS}_{\{k: 0 \leq k \leq 7\}}=\left\{\begin{array}{l}
0, \quad \text { if }, \beta\left(O_{k}^{q}\right)=0, \\
\alpha\left(\mathrm{G}_{k}\right) \times \beta\left(\mathrm{O}_{k}^{q}\right), \text { otherwise, }
\end{array}\right.
$$

where, $P S_{k}$ only generates scores at the $k^{\text {th }}$ neighbor when $\beta\left(O_{i}^{q}\right)$ is not zero. Thus, eq. (8) generates higher scores in the neighbors those form edge with center pixel. We are interested in $n$-neighbors having the maximum scores that are to be considered as the major directions of the inlying edge. In the coding, we distinguish these top $n$-directions by setting bit- 1 to their positions, while setting bit- 0 to others. Hence, the $L E P P$ code is defined as,

$$
L E P P_{n}=\sum_{k=0}^{7} Z_{k}\left(P S_{k}-P S_{n}\right) \times 2^{k}, \quad Z_{k}(a)=\left\{\begin{array}{l}
1, a \geq 0, \\
0, a<0,
\end{array}\right.
$$

where $P S_{n}$ is the $n$-th highest score. Fig. 2 shows an example of $L E P P$ code computation with $n=2$.

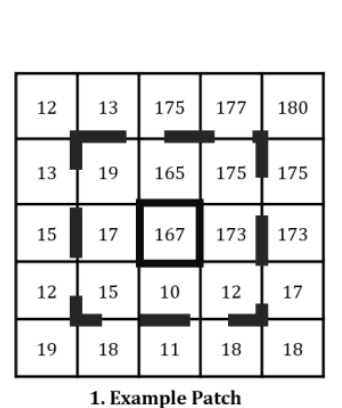

\begin{tabular}{|c|c|c|c|c|c|c|c|c|}
\hline Neighbor & 0 & $i$ & $i i$ & $i i i$ & iv & $v$ & $v i$ & $v i i$ \\
\hline Score & 132 & 0 & 132 & 0 & 0 & 0 & $\mathbf{0}$ & 61 \\
\hline Bit & 1 & 0 & 1 & 0 & 0 & $\mathbf{0}$ & $\mathbf{0}$ & 0 \\
\hline
\end{tabular}
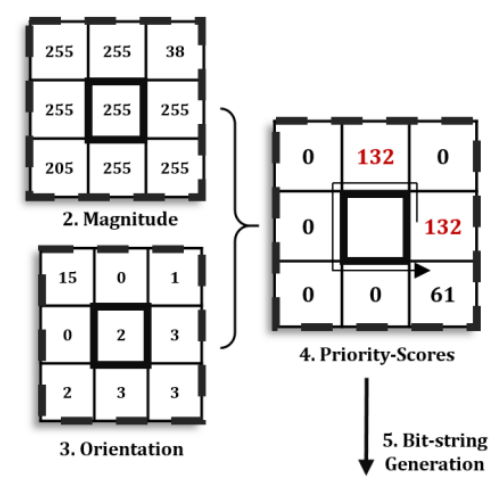

Fig.2. LEPP Code computation (with $n=2$ ) for the target pixel, marked in black box in the example patch.

\section{B. Feature-vector Generation}

We choose histogram-based approach to generate the final feature vector for each face. At each pixel, we compute $L E P P$ code according to eq. (9) and concatenate them into a single histogram to represent a facial description. To incorporate more locality in the code, we divide the image into more blocks $\left(R^{l}, R^{2}, \ldots ., R^{z}\right)$ and generate histogram for each block followed by concatenating them all into one feature vector.

$$
\begin{gathered}
H^{z}(\mathrm{k})=\sum_{(\mathrm{x}, \mathrm{y}) \in \mathrm{R}^{z}} \Delta(\mathrm{C}(\mathrm{x}, \mathrm{y}), \mathrm{k}), \Delta(a, b)=\left\{\begin{array}{l}
1, a=b, \\
0, a \neq b,
\end{array}\right. \\
C_{H}=\Omega_{z=1}^{m} H^{z},
\end{gathered}
$$

where, $C$ is the $L E P P$ code, $k$ is the number of histogram bin corresponding to a $C$ code of block $R^{z} . \Omega$ is concatenation operator that concatenates each individual histogram, $H^{z}$, computed from all $z=1,2, \ldots, n$ blocks, and forms single histogram, $C_{H}$. We regard $C_{H}$ as the final feature-vector in our approach, which we send to the classifier for the classification task.

\section{Discussion}

In this section, we discuss some of the key advantages of the proposed method in preserving diverse variations of aging-affiliated textures.

One key advantage of LEPP is that it represents the structure of the different wrinkle patterns, appropriately. To show this, we provide sample-patches of a stronger and a smoother wrinkle in Fig. 3. Both the wrinkles possess vertical edges, and we observe that LEPP successfully identifies the proper direction of the edges by generating maximum priority-scores at the vertical directions (at position $i i$ and $v i$ ), respectively. This is especially significant for the smoother wrinkles since existing descriptors often misinterpret smoother wrinkles as other random-textures (e.g., flat texture) owing to the inability to characterize the gradual texture-change of such wrinkles. Nevertheless, LEPP explores wider region than that of the other edge-descriptors, and gain richer knowledge of the neighboring structure in order to characterize the gradual texture change information of smoother wrinkles, generating distinctive code for them. In this regard, we also note that LEPP differentiates the textures from featureless flat region. We show such an example in Fig. 3, where we point that LEPP applies a threshold on the magnitude of the pixel in order to filter such texture. Pixels having a lower magnitude (than the threshold), are not taken into account in the codegeneration, afterwards.

Using such wider neighboring information is also advantageous to be more stable against slight distortion and subtle noise-effect in local region. Therefore, LEPP is able to generate consistent code-pattern in the continuing wrinkles. To make it more elaborate, we 

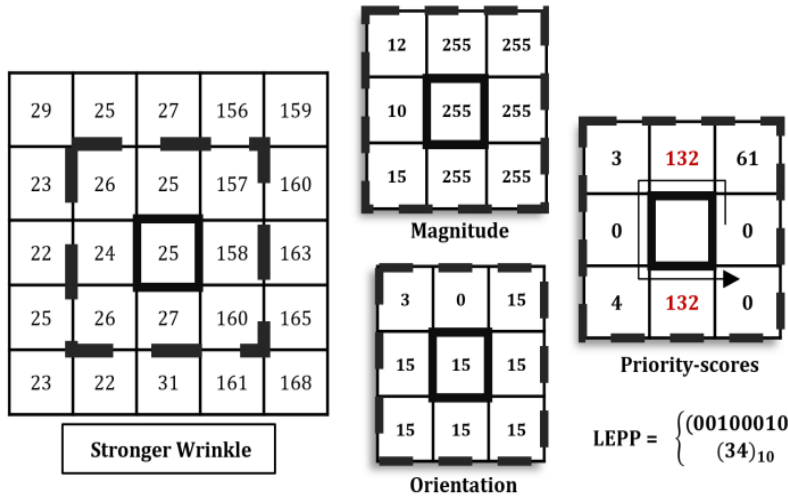

LEPP $=\left\{(00100010)_{2}\right.$
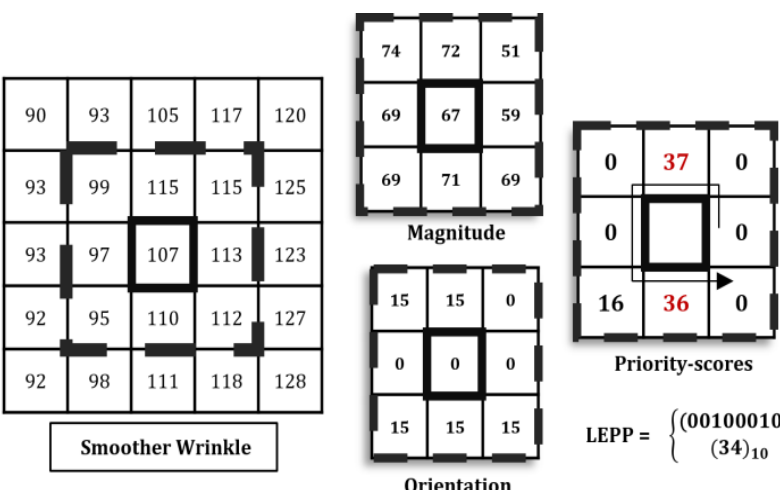

Priority-scores

LEPP $=\left\{(00100010)_{2}\right.$

Orientation
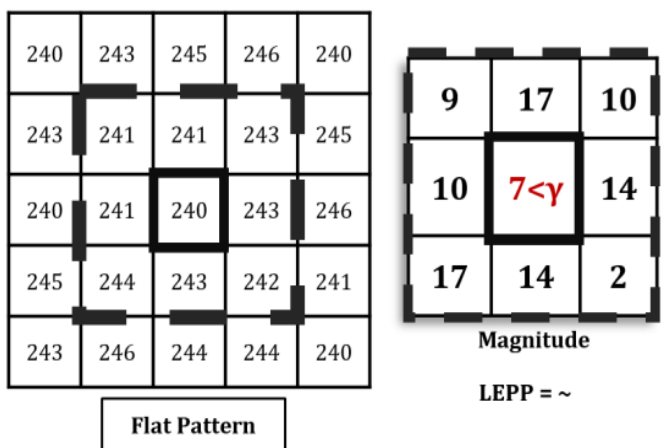

LEPP $=\sim$

Fig.3. LEPP code for a stronger wrinkle, smoother wrinkle, and a flat patch. We use $\gamma=20$ as the threshold.

present an example Fig. 4, where we show the generation of LEPP codes for different parts of a continuing wrinkle. For this, we aim to generate codes at some specific parts of the target wrinkle, where different types of edges are evident, such as diagonal-edge, horizontal-edge, and a curved-edge. We observe that despite having uneven intensity variations around the wrinkle, the diagonal, horizontal, and curved directions are characterized successfully by LEPP, which can be understood by observing maximum priority-scores generated at the diagonal, horizontal and curved positions, respectively. These examples strongly demonstrate the efficacy of LEPP in generating stable wrinkle codes under subtle local distortions.

\section{EXPERIMENTAL RESULTS}

In this section, we present a comprehensive experimental analysis of LEPP regarding its selection of optimal parameters, recognition accuracy in popular datasets, its stability under noise, and its computation time. We perform age-group recognition experiments in three datasets, including, ADIENCE [26], GALLAGHER [33], and FACES dataset [34].

\section{A. Experimental Settings}

We start our experiment by cropping the face from input images using the given landmark position or manual selection, followed by normalizing it to $110 \times 150$ pixels. As described, we divide the face into several uniform non-overlapping blocks and concatenate all the code histograms from these blocks to generate the final feature-vector. For classification, we apply Support Vector Machine (SVM) [35] with RBF kernel function since it has already shown promising result in appearance-based age recognition [27]-[28]. However, to select the optimal parameters, we conduct a grid-search on the hyper-parameters within a cross-validation approach and pick the parameter-values giving the best cross-validation results.

To generate the recognition results for LEPP, we conduct standard 5-fold subject-exclusive crossvalidation experiments, as done in previous works [22], [23], [26], [28]. Subject-exclusive folds are generated in such a way that the folds are disjoint, that is, a subject appearing in one fold does not appear in another fold.

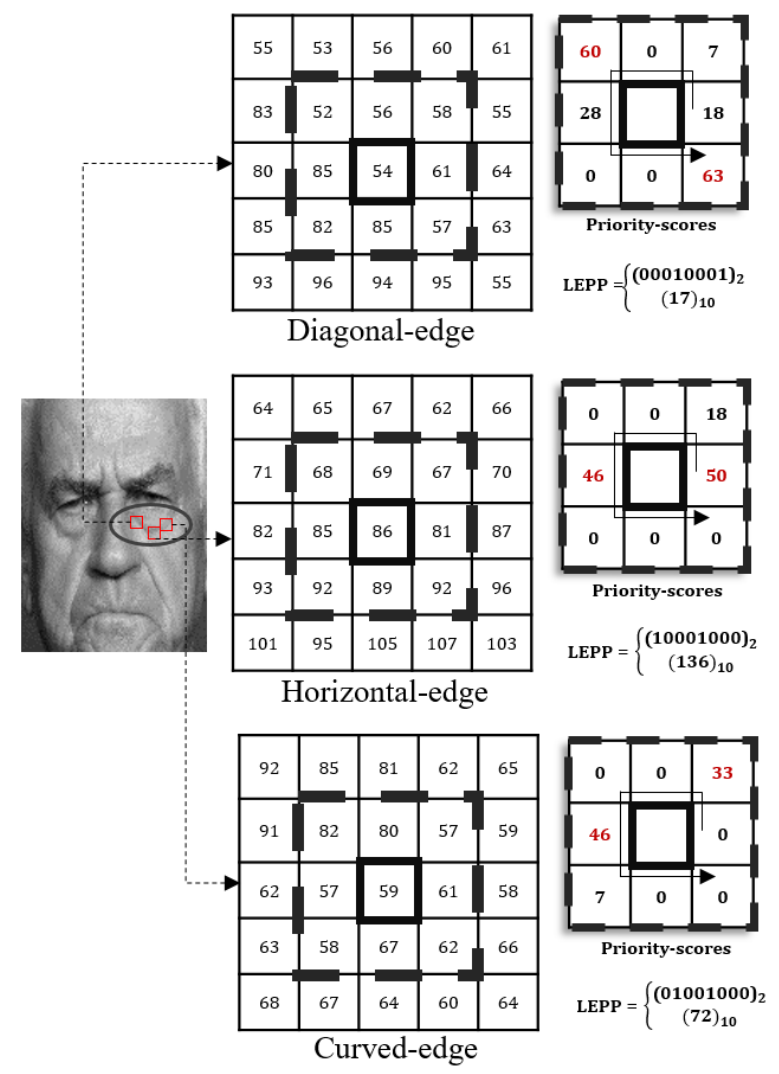

Fig.4. LEPP code in different parts of a wrinkle, especially in a diagonal-edge, a horizontal-edge and a curved-edge. 


\section{B. ADIENCE Results}

ADIENCE [26] is a recently published age-group dataset including both male and female face images within 8 age groups $(0-2,4-6,8-13,15-20,25-32,38-43$, $48-53,60+$ ). ADIENCE dataset consists of real-world facial images appearing at significant rotation, expression and pose variations. However, there are two subsets available in this dataset: frontal and complete set. Similar to [26], [28], we conduct experiments on frontal set in this work.

Selection of Optimal Parameters: There are several parameters of the proposed method, such as, threshold $\gamma$ (in eq. (5)), weight-values for $\eta$ and $\xi$ (in eq. (8)), the number of edge-direction $n$ (in eq. (9)), and block-size (in eq. (10)), which need to be set. For the parameter selection purpose, we test the performance of the proposed method for different parameter-values in ADIENCE. The values giving the best recognition rate are selected as the optimal parameters.

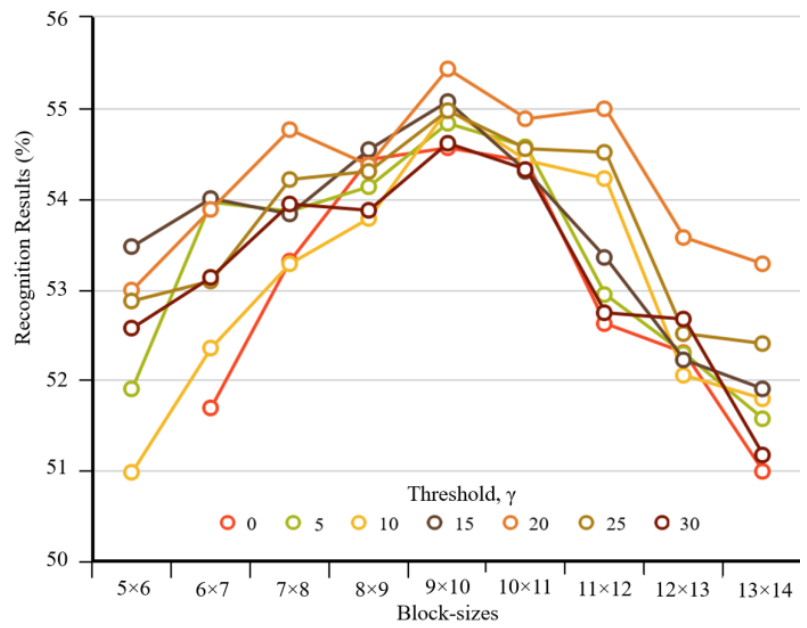

Fig.5. Performance of LEPP for different threshold values and blocksizes in ADIENCE.

At first, we test the performance of LEPP for different $\gamma$ values varying from 0 to 30 with a stride of 5 within different block sizes varying from $5 \times 6$ to $13 \times 14$. The results are shown in Fig. 5. We observe that the highest result is achieved at considering $\gamma=20$ and a block-size of $9 \times 10$. We note that a higher threshold-value may eliminate number of pixels from the image. Therefore, while generating a histogram of a smaller-sized block, one may face lack of samples, causing sampling-error, in practice [36]. Moreover, setting a lower threshold keeps a considerable amount of flat pixels in the face image, which may add redundant information in the feature description. On the contrary, considering comparably larger-sized blocks may possess limited spatial information. Therefore, $\gamma=20$ and block-size of $9 \times 10$ gives a good trade-off between preserving sufficient spatial information and adequate samples, and hence we consider these values as the optimal parameters.

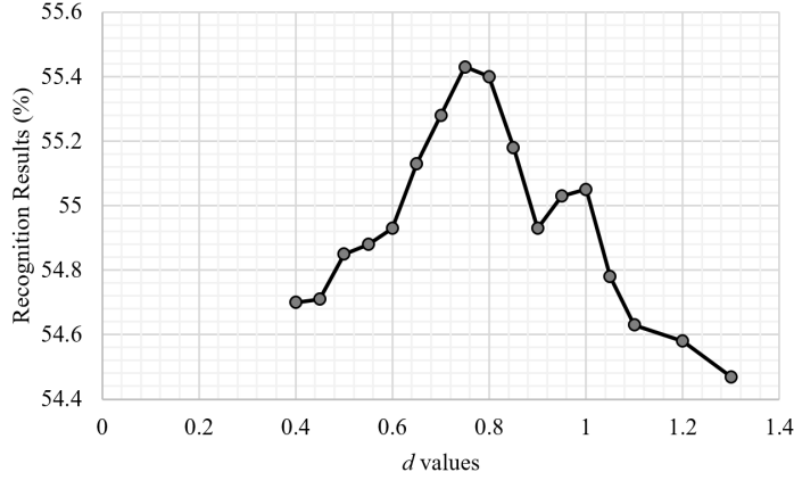

Fig.6. Performance of LEPP in ADIENCE for different standard deviation, $d$, in setting the Gaussian weight

Moreover, as mentioned earlier, we set the value of $\eta$ and $\xi$ through Gaussian weighting with zero-mean and standard deviation, $d$. That is, different value of standard deviation $d$ can generate different values of $\eta$ and $\xi$. Hence, to select the optimal $d$ value, we test LEPP with different $d$ values ranging from 0.4 to 1.3 . Fig. 6 shows that the highest result is achieved at $d=0.75$. However, using $d=0.75$ with standard Gaussian formula, we get the value of $\eta$ and $\xi$ as 0.518612 and 0.240694 , respectively. This means that the higher weight $\eta$ is almost double of the lower weight $\xi$, which appropriately serves our purpose in prioritizing the target neighbors. Therefore, we consider $d=0.75$ as the optimal standard deviation value in setting the value of $\eta$ and $\xi$.

Selection of the direction of edge, $n$, is another important factor in LEPP. For this, we test LEPP for different $n$ values and present the results at Fig. 7, where we observe the highest result at considering $n=2$. Considering $n=1$, LEPP achieves the worst result. However, $n=1$ points to use only the primary edge direction of the pixel, which may miss the other edge directions that are required for corner and curve-like textures. In this regard, $n=2$ is more suitable to represent such texture structure with its two edge direction, which is also evident in the results presented at Fig. 6. On the contrary, $n=3$ is usually appropriate for complex junction-like edge structure. However, the facial image does not possess such complex structure, which why it

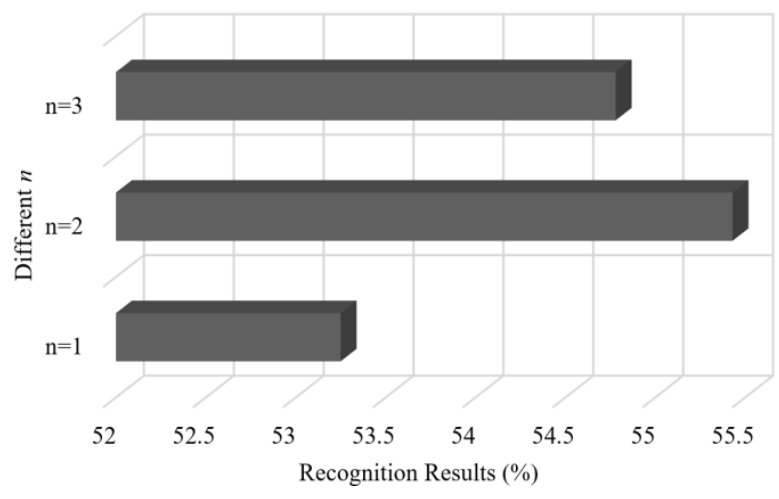

Fig.7. Performance of LEPP for different edge-direction, $n$, in ADIENCE. 
may often add redundant information to the pixel structure, contributing towards its inferior accuracy than that of $n=2$. Therefore, we consider $n=2$ for the optimal $n$ value for $L E P P$.

Performance under Noise: We evaluate the performance of LEPP under noisy environment in order to evaluate its stability against such condition. For this purpose, we artificially add random Gaussian noise with zero mean and standard deviation within two intervals $(0.08 \sim 0.16$ and $0.16 \sim 0.32)$ for each image of ADIENCE dataset. It is noteworthy to mention that noises within the given intervals are randomly distributed in order to cope with the uneven natural condition. We now evaluate the recognition accuracy of LEPP against other descriptors, including LBP, LDP, LDN, PTP, and DAPP with 5-fold subject-exclusive cross-validation protocol, and present the results in Table 1. Note that we also present results without adding any noise, in this table. However, for both the cases, proposed descriptor achieves better accuracies than other descriptors, exhibiting its efficacy under such noisy condition. One key reason behind this consistent performance is that LEPP uses larger neighboring area in order to encode the feature-description, which allows LEPP to tackle the effect of subtle noise and distortion of intensity within local texture yielding the generation of stable feature codes. Moreover, some featureless textures, such as flat patterns may change their structure easily under noise. LEPP applies a threshold to filter such uncertain featureless pixels. Therefore, the effect of the uncertain patterns is also limited in LEPP, contributing towards its consistent performance.

Table 1. Performance of Different Descriptors under Noise in ADIENCE dataset

\begin{tabular}{|c|c|c|c|}
\hline \multirow{2}{*}{ Descriptors } & \multirow{2}{*}{ No noise } & \multicolumn{2}{|c|}{ Noise Variances } \\
\cline { 3 - 4 } & & $\mathbf{0 . 0 8} \sim \mathbf{0 . 1 6}$ & $\mathbf{0 . 1 6} \mathbf{0 . 3 2}$ \\
\hline LBP & $41.1[26]$ & 31.06 & 28.98 \\
\hline LDP & $48.5[26]$ & 35.44 & 32.21 \\
\hline LDN & $51.42[28]$ & 36.35 & 33.75 \\
\hline PTP & $53.27[28]$ & 39.55 & 35.87 \\
\hline DAPP & $54.9[28]$ & 40.67 & 38.64 \\
\hline LEPP & $\mathbf{5 5 . 4 3}$ & $\mathbf{4 3 . 8 7}$ & $\mathbf{4 0 . 0 8}$ \\
\hline
\end{tabular}

Note: Results with citations are from the original paper

Table 2. Comparative Recognition Results against state-of-the-art Results in ADIENCE dataset with 5-fold subsect exclusive protocol

\begin{tabular}{|c|c|}
\hline Methods & Results (\%) \\
\hline LBP [26] & 41.1 \\
\hline FPLBP [26] & 39.8 \\
\hline LBP+FLBP [26] & 44.5 \\
\hline LBP+FPLBP+P0.5 [26] & 38.1 \\
\hline LBP+FPLBP+P0.8 [26] & 32.9 \\
\hline LBP+FPLBP+D0.5 [26] & 44.5 \\
\hline LBP+FPLBP+D0.8 [26] & 45.1 \\
\hline LDP [28] & 48.5 \\
\hline LDN [28] & 51.42 \\
\hline Best from [23] & 50.7 \\
\hline Cascaded DCNN [22] & 52.88 \\
\hline PTP [28] & 53.27 \\
\hline DAPP [28] & 54.9 \\
\hline LEPP & $\mathbf{5 5 . 4 3}$ \\
\hline Note: Results with citations are from the original paper
\end{tabular}

Recognition Accuracy: We test the performance of LEPP in ADIENCE dataset within predefined eight age groups. Table 2 present the results for 5-fold subjectexclusive protocol. Results show that LEPP outperforms the existing descriptors, including LBP, FPLLBP, LDP, LDN, PTP and recently published DAPP. Moreover, it is important to notice that, LEPP performs better than contemporary deep learning methods [22]-[23]. The possible reason behind this better accuracy than the deepmethods is that LEPP preserves the appropriate shape of wrinkle, which enables clear aging definition in the classification. Moreover, LEPP discards the uncertain featureless codes from its feature-vector, which ensures the sole presence of specific aging-affiliated textured code in the histogram. Such explicit representation of aging-affiliated feature perhaps works better than the higher-level features generated by deep learning methods, contributing to the higher accuracy of LEPP.

\section{GALLAGHER Results}

Gallagher dataset [33] consists of a collection of people images with seven age categories: $0-2,3-7,8-12$, 13-19, 20-36, 37-65, and 66+. Images are collected from various social programs photos, like, wedding, family programs, naïve group photos, etc. GALLAGHER images preserve considerable variability in the images due to the natural face images. For instance, people having dark glasses, face occlusions, and unusual facial expressions can be found in the images, which makes the dataset very challenging in nature.

Table 3. Comparative Recognition Results against state-of-the-art Results in GALLAGHER dataset with 5-fold subsect exclusive protocol

\begin{tabular}{|c|c|}
\hline Methods & Results (\%) \\
\hline LBP [26] & 58.0 \\
\hline FPLBP [26] & 61.0 \\
\hline LBP+FLBP [26] & 59.0 \\
\hline LBP+FPLBP+P0.5 [26] & 46.8 \\
\hline LBP+FPLBP+P0.8 [26] & 41.3 \\
\hline LBP+FPLBP+D0.5 [26] & 64.3 \\
\hline LBP+FPLBP+D0.8 [26] & 66.6 \\
\hline LDP [28] & 66.8 \\
\hline LDN [28] & 67.4 \\
\hline PTP [28] & 68.8 \\
\hline DAPP [28] & 69.91 \\
\hline LEPP & $\mathbf{7 0 . 4 4}$ \\
\hline
\end{tabular}

Note: Results with citations are from the original paper

Table 3 shows the comparative results of LEPP in GALLAGHER dataset. From the presented result, we observe the preeminence of LEPP over existing descriptors. It is important to notice that the results of LEPP are comparatively closer to the edge-based descriptors, such as PTP and DAPP. Nonetheless, the result of LEPP is considerably better than the texturebased approaches like LBP, FPLBP, and their fusions, showing its superiority over such approaches.

However, some of the unsuccessful predictions are given in Fig. 8. 


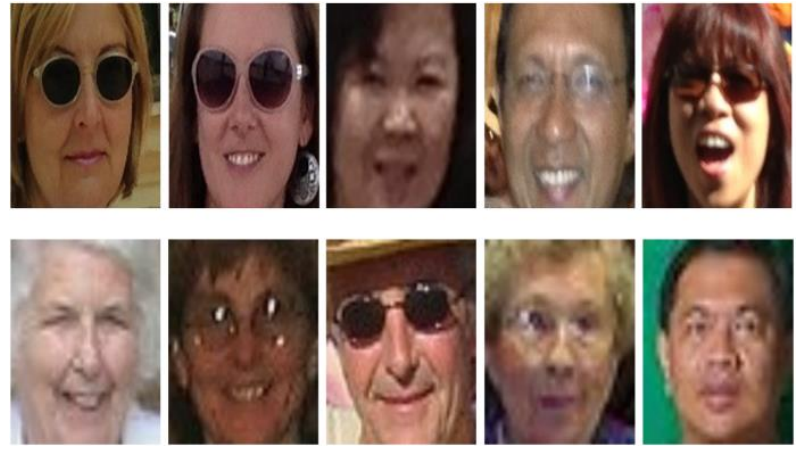

Fig.8. Some unsuccessful predictions from ADIENCE (upper row) and GALLAGHER (lower row) datasets

\section{FACES Results}

FACES dataset [34] has three image groups: young, middle-aged, and older. Among them, there consists 61 young subjects, 60 middle-aged subject, and 58 older subjects. FACES images are of different varieties, comprising male, female and different expressions. The images were taken under controlled studio environment, and images were carefully post-processed after being taken.

We generate the result for LEPP and other descriptors among three age groups in FACES dataset. We present the results in Table 4, where LEPP shows better performance than LBP, LDP, LDN, PTP, and DAPP. However, it is interesting to note that, LBP performs better than some edge-based descriptors, such as LDP, LDN, and PTP. Perhaps this happens because of the controlled nature of the FACES images, where the effect of noise and uneven illumination are trivial. We also note that the overall recognition accuracies are high in FACES compared to former two datasets owing to the less number of classes in FACES. Moreover, the controlled nature of the face images that avoid the natural deviation of head, lip, and other noise-affect, also contribute towards such overall higher recognition accuracies in FACES dataset. However, the reason for the high accuracy of LEPP than other descriptors in FACES is straightforward. Typically, the older face images possess a high amount of wrinkles. Since LEPP appropriately depicts the wrinkle structure, LEPP feature-histogram contains diverse wrinkle codes. On the contrary, infant and young aged faces possess fewer wrinkles while having more regions that are flat. LEPP explicitly discards the flat patterns in the coding, and hence, infant's faces possess fewer accumulations of wrinkle codes compared to older faces, which clearly differentiates these images from the older one.

Table 4. Comparative Recognition Results in FACES dataset with 5fold subsect exclusive protocol

\begin{tabular}{|c|c|}
\hline Methods & Results (\%) \\
\hline LBP & 83.3 \\
\hline LDP & 82.0 \\
\hline LDN & 82.5 \\
\hline PTP & 78.31 \\
\hline DAPP & 83.43 \\
\hline LEPP & $\mathbf{8 4 . 2 5}$ \\
\hline
\end{tabular}

\section{Computation Time}

We show the computation time of proposed LEPP in terms of the average time to compute its feature-vector. We conduct this experiment with Visual Studio 2015 on Intel Core i5 @2.67GHz with 8GB RAM. For this experiment, we randomly collect 100 images from the working datasets and record the time required for extracting LEPP feature-vector for each of the images. We compute the average time in milliseconds (ms), and present it in Table 5. We also conduct the same procedure to calculate the computation-time for other descriptors. It is evident that LBP is computationally most efficient method among others owing to its computational simplicity. Edge descriptors, including LDP, LDN, LPTP, and PTP require comparatively more time than LBP due to the convolution process of compass masks. However, proposed LEPP requires slightly more time than more time than the above-mentioned edge-descriptors. For each target pixel, LEPP has to check all the neighbors with its respective structural restrictions, which perhaps consume considerable computation, contributing towards such extra time. However, LEPP still achieves higher accuracy than recently published DAPP, and most importantly, it provides us significant gain in recognition results along with its stable performance under noise. Such stable recognition accuracy, robust performance and moderate computation time strongly advocate the efficacy of LEPP in the recognition of age groups in multi-disciplinary purpose.

Table 5. Feature-vector Computation Time of Different Descriptors

\begin{tabular}{|c|c|}
\hline Descriptors & Computation Time (ms) \\
\hline LBP & 3.5 \\
\hline LDP & 4.8 \\
\hline LDN & 4.6 \\
\hline PTP & 4.4 \\
\hline DAPP & 6.7 \\
\hline LEPP & 6.1 \\
\hline
\end{tabular}

\section{CONCLUSION}

In this paper, we deal age recognition problem signifying the importance of characterizing diverse wrinkle patterns with consistent feature-codes and propose a new feature descriptor, LEPP, to address this issue. Proposed LEPP descriptor characterizes the inlying edge shape of the pixel by prioritizing the neighbors that form an edge with the center pixel. LEPP initiates a set of structural restrictions to prioritize the neighboring pixels, through which, it avoids the random patterns and represents the appropriate direction of the inlying edge of the wrinkles. Moreover, LEPP uses a wider neighboring area to generate the code, which allows LEPP to be stable against subtle local distortion and noise. We evaluable the performance of LEPP in three benchmark datasets, where LEPP clearly shows its superiority over existing descriptors.

However, extending the proposed coding scheme of LEPP for other facial analysis tasks, such as age estimation, expression and gender recognition, can be 
regarded as potential future works. We leave these here as our future endeavors.

\section{REFERENCES}

[1] Y. Fu, G. Guo, and T. S. Huang. Age synthesis and estimation via faces: A survey. Pattern Analysis and Machine Intelligence, IEEE Transactions on, 32(11):1955-1976, 2010.

[2] N. Ramanathan, R. Chellappa, and S. Biswas. Computational methods for modeling facial aging: A survey. Journal of Visual Languages \& Computing, 20(3):131-144, 2009.

[3] Y. Fu and T. S. Huang. Human age estimation with regression on discriminative aging manifold. Multimedia, IEEE Transactions on, 10(4):578-584, 2008. 1

[4] N. Ramanathan and R. Chellappa. Face verification across age progression. IEEE Transactions on Image Processing, 15(11):3349-3361, 2006.

[5] A.M. Albert, K. Ricanek, Jr., and E. Patterson, "A review of the literature on the aging adult skull and face: Implications for forensic science research and applications," Forensic Sci. Int., vol. 172, no. 1, pp. 1-9, 2007.

[6] M. Albert, A. Sethuram, and K. Ricanek, Implications of Adult Facial Aging on Biometrics. Rijeka, Croatia: InTech, 2011.

[7] E. Patterson, A. Sethuram, M. Albert, K. Ricanek, and M. King, "Aspects of age variation in facial morphology affecting biometrics," in Proc. 1st IEEE Int. Conf. Biometrics, Theory, Appl., Syst. (BTAS), Sep. 2007, pp. $1-6$.

[8] A. Lanitis, "A survey of the effects of aging on biometric identity verification," Int. J. Biometrics, vol. 2, no. 1, pp. 34-52, Dec. 2010.

[9] Y. Bando, T. Kuratate, and T. Nishita. A simple method for modeling wrinkles on human skin. In Computer Graphics and Applications, 2002. Proceedings. 10th Pacific Conference on, pages 166-175. IEEE, 2002.

[10] B. Tiddeman, M. Burt, and D. Perrett. Prototyping and transforming facial textures for perception research. IEEE computer graphics and applications, 21(5):42-50, 2001.

[11] Y. Wu, P. Kalra, L. Moccozet, and N. MagnenatThalmann. Simulating wrinkles and skin aging. The visual computer, 15(4):183-198, 1999

[12] S. E. Choi, Y. J. Lee, S. J. Lee, K. R. Park, and J. Kim. Age estimation using a hierarchical classifier based on global and local facial features. Pattern Recognition, 44(6):1262-1281, 2011.

[13] M. M. Dehshibi and A. Bastanfard. A new algorithm for age recognition from facial images. Signal Processing, 90(8):2431-2444, 2010

[14] M. A. Hajizadeh and H. Ebrahimnezhad. Classification of age groups from facial image using histograms of oriented gradients. In Machine Vision and Image Processing (MVIP), 2011 7th Iranian, pages 1-5. IEEE, 2011

[15] J.-i. Hayashi, M. Yasumoto, H. Ito, and H. Koshimizu. Age and gender estimation based on wrinkle texture and color of facial images. In Pattern Recognition, 2002. Proceedings. 16th International Conference on, volume 1, pages 405-408. IEEE, 2002

[16] W.-B. Horng, C.-P. Lee, and C.-W. Chen. Classification of age groups based on facial features. Tamkang Journal of Science and Engineering, 4(3):183-192, 2001

[17] A. Lanitis, C. J. Taylor, and T. F. Cootes. Modeling the process of ageing in face images. In Computer Vision,
1999. The Proceedings of the Seventh IEEE International Conference on, volume 1, pages 131-136. IEEE, 1999

[18] K. Luu, K. Ricanek Jr, T. D. Bui, and C. Y. Suen. Age estimation using active appearance models and support vector machine regression. In Biometrics: Theory, Applications, and Systems, 2009. BTAS'09. IEEE 3rd International conference on, pages 1-5. IEEE, 2009

[19] X. Geng, Z.-H. Zhou, and K. Smith-Miles. Automatic age estimation based on facial aging patterns. Pattern Analysis and Machine Intelligence, IEEE Transactions on, 29(12):2234-2240, 2007.

[20] G. Guo, Y. Fu, C. R. Dyer, and T. S. Huang. Image-based human age estimation by manifold learning and locally adjusted robust regression. Image Processing, IEEE Transactions on, 17(7):1178-1188, 2008

[21] J. Lu and Y.-P. Tan. Ordinary preserving manifold analysis for human age and head pose estimation. HumanMachine Systems, IEEE Transactions on, 43(2):249-258, 2013.

[22] J.-C. Chen, A. Kumar, R. Ranjan, V. M. Patel, A. Alavi, and R. Chellappa. A cascaded convolutional neural network for age estimation of unconstrained faces. In Biometrics Theory Applications and Systems (BTAS), 2016 IEEE 8th International Conference on, pages 1-8. IEEE, 2016.

[23] G. Levi and T. Hassner. Age and gender classification using convolutional neural networks. In Proceedings of the IEEE Conference on Computer Vision and Pattern Recognition Workshops, pages 34-42, 2015.

[24] G"unay and V. V. Nabiyev. Automatic age classification with lbp. In Computer and Information Sciences, 2008. ISCIS' 08. 23rd International Symposium on, pages 1-4. IEEE, 2008.

[25] J. Ylioinas, A. Hadid, and M. Pietikainen. Age classification in unconstrained conditions using lbp variants. In Pattern Recognition (ICPR), 2012 21st International Conference on, pages 1257-1260. IEEE, 2012

[26] E. Eidinger, R. Enbar, and T. Hassner. Age and gender estimation of unfiltered faces. IEEE Transactions on Information Forensics and Security, 9(12):2170-2179, 2014

[27] M. T. B. Iqbal, B. Ryu, G. Song, and O. Chae. Positional ternary pattern (ptp): An edge based image descriptor for human age recognition. In 2016 IEEE International Conference on Consumer Electronics (ICCE), pages 289292, IEEE, 2016.

[28] M. T. B. Iqbal, M. Shoyaib, B. Ryu, M. Abdullah-AlWadud, and O. Chae. "Directional age-primitive pattern (DAPP) for human age group recognition and age estimation." IEEE Transactions on Information Forensics and Security 12.11 (2017): 2505-2517.

[29] T. Jabid, M. H. Kabir, and O. Chae. Robust facial expression recognition based on local directional pattern. ETRI journal, 32(5):784-794, 2010

[30] A. Ramirez Rivera, J. Rojas Castillo, and O. Chae. Local directional number pattern for face analysis: Face and expression recognition. Image Processing, IEEE Transactions on, 22(5):1740-1752, 2013.

[31] A. C. Bovik, Handbook of image and video processing. Academic press, 2010.

[32] L. Shapiro, Computer vision and image processing. Academic Press, 1992.

[33] A. C. Gallagher and T. Chen. Understanding images of groups of people. In Computer Vision and Pattern Recognition, 2009. CVPR 2009. IEEE Conference on, pages 256-263. IEEE, 2009. 
[34] N. C. Ebner, M. Riediger, and U. Lindenberger. Faces-A database of facial expressions in young, middle-aged, and older women and men: Development and validation. Behavior research methods, 42(1):351-362, 2010.

[35] C.-W. Hsu and C.-J. Lin, "A comparison of methods for multiclass support vector machines," IEEE Trans. Neural Netw., vol. 13, no. 2, pp. 415-425, Mar. 2002

[36] J. Ylioinas, A. Hadid, Y. Guo, and M. Pietikäinen, "Efficient image appearance description using dense sampling based local binary patterns," in Computer Vision. Berlin, Germany: Springer, 2013, pp. 375-388.

\section{Authors' Profiles}

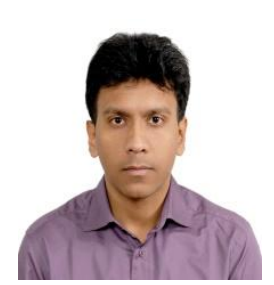

Md Tauhid Bin Iqbal received his Bachelor degree in Information Technology from University of Dhaka in 2012. Currently he is pursuing MS-Ph.D. combined degree in Department of Computer Science \& Engineering, Kyung Hee University, Yongin-si, Gyeonggi-do, South Korea. His current research interests include pattern recognition, object detection, expression recognition, combined age $\&$ gender recognition.

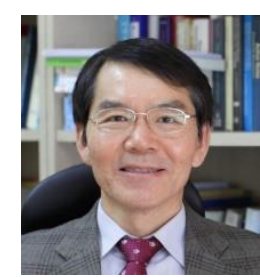

Oksam Chae (M92) received the B.Sc. degree in electronics engineering from Inha University, Incheon, South Korea, in 1977, and the M.S. and Ph.D. degrees in electrical and computer engineering from Oklahoma State University, Stillwater, in 1982 and 1986, respectively. He was a Research Engineer with Texas Instruments Image Processing Laboratory, Dallas, TX, from 1986 to 1988. Since 1988, he has been a Professor with the Department of Computer Engineering, KyungHee University, Gyeonggido, South Korea. His current research interests include multimedia data processing environments, intrusion detection systems, sensor networks, and medical image processing in dentistry. Prof. Chae is a member of the SPIE, Korean Electronic Society (KES), and the Institute of Electronics, Information and Communication Engineers.

How to cite this paper: Md Tauhid Bin Iqbal, Oksam Chae, " Mining Wrinkle-Patterns with Local Edge-Prototypic Pattern (LEPP) Descriptor for the Recognition of Human Age-groups ", International Journal of Image, Graphics and Signal Processing(IJIGSP), Vol.10, No.7, pp. 1-10, 2018.DOI: 10.5815/ijigsp.2018.07.01 